\title{
Botanica Complutensis
}

ISSN-e: 1988-2874

\section{Bibliografía Botánica Ibérica, 2017. Fungi}

Ana Rosa Burgaz ${ }^{1}$

Cómo citar: Burgaz, A. R. (2018). Bibliografía Botánica Ibérica, 2017. Fungi. Bot. complut. 42: 175-179.

2719. Ballarà, J. 2017. Recolecciones de Cortinarius subserratissimus en la Península Ibérica verificadas con soporte de la secuenciación. Yesca 29: 119127. (SisM, Cortinarius, B).

2720. Bellido, F., Moreno, G., Meyer, M. \& Castillo, A. 2017. A new species of Didymium from Spain. Bol. Soc. Micol. Madrid 41: 17-22. (Tax, Didymium, Av, H).

2721. Burgaz, A.R. 2017. Bibliografía Botánica Ibérica, 2016. Fungi. Bot. Complut. 41: 101-107. (Bibl).

2722. Caballero, F., Alvarado, P. \& Fernández-Brime, S. 2017. Contribución al conocimiento de la micoflora del Parc de la Serralada litoral. Rev. Cat. Micol. 38: 71-94. (Flora, Basidiomycetes, B).

2723. Calle, J.R. 2017. Algunos comentarios sobre la ecología de Hypholoma fasciculare. Yesca 29: 51-56. (Ecol, Hypholoma, $\mathrm{S})$.

2724. Corral, S. 2017. Myriostoma coliforme (Dicks.) Corda en el Morrazo. Yesca 29: 111-118. (Flora, Myriostoma, Po).

2725. Crous, P.W. et al. 2017. Coniella heterospora Valenzuela-López, Cano, Guarro \& Stchigel, sp. nov. Persoonia. Fungal Planet description sheets: 625-715. 39: 290-291. (SisM, Coniella, H). https://doi.org/10.3767/persoonia.2017.39.11.

2726. Crous, P.W. et al. 2017. Hymenochaete macrochloae Olariaga \& $\mathrm{M}$. Prieto, sp. nov. Persoonia. Fungal Planet description sheets: 625-715. 39: 308-309. (SisM, Hymenochaete, M, To, SS). https://doi.org/10.3767/persoonia.2017.39.11.
2727. Crous, P.W. et al. 2017. Ramaria cistophila P.P. Daniëls, M.P. Martín, C. Rojo \& Camello, sp. nov. Persoonia. Fungal Planet description sheets: 625715. 39: 335-336. (SisM, Ramaria, Ca, Cc, Za). https://doi.org/10.3767/persoonia.2017.39.11.

2728. Crous, P.W. et al. 2017. Coprinopsis pseudomarcescibilis Heykoop, G. Moreno \& P. Alvarado, sp. nov. Persoonia. Fungal Planet description sheets: 558-624. 38: 320-321. (SisM, Coprinopsis, Gu). doi: https://doi. org/10.3767/003158517X698941.

2729. Crous, P.W.etal. 2017. Gyroporuspseudocyanescens G. Moreno, Carlavilla, Heykoop, Manjón\&Vizzini, sp. nov. Persoonia. Fungal Planet description sheets: 558-624. 38: 328-329. (SisM, Gyroporus, Cc, Gu, O, Sg). doi: https:// doi.org/10.3767/003158517X698941.

2730. Crous, P.W. et al. 2017. Inocybe parvicystis F.J. Rodr.-Campo \& EsteveRav., sp. nov. Persoonia. Fungal Planet description sheets: 558-624. 38: 338339. (SisM, Inocybe, M). doi: https:// doi.org/10.3767/003158517X698941.

2731. Crous, P.W. et al. 2017. Penicillium parvofructum Guevara-Suarez, Cano-Canals, Cano \& Stchigel, sp. nov. Persoonia. Fungal Planet description sheets: 558-624. 38: 352-353. (SisM, Penicillium, T). doi: https://doi. org/10.3767/003158517X698941.

2732. De Esteban-Resino, J. \& Pérez-Gorjón, S. 2017. Hyphoderma deviatum (Meruliaceae, Basidiomycota) en la Península Ibérica. Bol. Micol. FAM-

1 Departamento de Biodiversidad, Ecología y Evolución (Unidad de Botánica), Facultad de Biología, Universidad Complutense de Madrid. e-mail: arburgaz@ucm.es

http://dx.doi.org/10.5209/BOCM.61376 
CAL 12: 61-65. (Tax, Hyphoderma, Phlebia, Sa, To).

2733. Díaz Fernández, A., Rodea Butragueño, J.A. \& Rodríguez-Campo, F.J. 2017. Ionomidotis irregularis (Schwein.) E.J. Durand (1923) Un raro ascomycete encontrado en Saja (Cantabria). Yesca 29: 85-92. (Flora, Inomidotis, S).

2734. Eiroa García-Garabal, J.A., Eiroa Rosado, E. 2017. Un ataque por Serpula lacrimans. Yesca 29: 41-46. (Ecol, Serpula, C).

2735. Fernández-Miranda Cagigal, E. \& Casares Sánchez, A. 2017. Estructura, biogénesis y dependencia nutritiva de los esclerocios de Paxillus ammoniavirescens (Boletales, Paxillaceae). Anales Jard. Bot. Madrid 74(1): e054 2017. ISSN: 0211-1322. (Anat, Paxillus). doi: http://dx.doi.org/10.3989/ ajbm. 2454.

2736. García-Barreda, S., Molina-Grau, S., Forcadell, R., Sánchez, S. \& Reyna, S. 2017. Long-term soil alteration in historical charcoal hearths affects $\mathrm{Tu}$ ber melanosporum mycorrhizal development and environmental conditions for fruiting. Mycorrhiza 27(6): 603609. (Ecol, Quim, Tuber, Cs, Te). doi: 10.1007/s00572-017-0773-0.

2737. Giraldo, A., Crous, P.W., Schumacher, R.K., Cheewangkoon, R., Ghobad-Nejhad, M. \& Langer, E. 2017. The genera of fungi-G3: Aleurocystis, Blastacervulus, Clypeophysalospora, Licrostroma, Neohendersonia and Spumatoria. Mycol. Progress 16(4): 325-348. (SisM, Clypeophysalospora, Portugal). doi 10.1007/s11557-017-1270-8.

2738. González-Menéndez, V., Martin, J., Siles, J.A., González-Tejero, M. R., Reyes, F., Platas, G., Tormo, J.R. \& Genilloud, O. 2017. Biodiversity and chemotaxonomy of Preussia isolates from the Iberian Peninsula. Mycol. Progress 16(7): 713-728. (SisM, Preussia, Esp). doi: 10.1007/s11557-017-1305-1.

2739. Gorjón, S.P.\&Bernicchia,A.2017.Dendrocorticium pinsapineum (Corticiales, Basidiomycota), second world distributional area in Italy. Nova Hedwigia 105(3-4): 341-346. (Tax Dendrocorticium, Ma). doi: https://doi.org/10.1127/ nova_hedwigia/2017/0415.
2740. Heykoop, M., Moreno, G., Alvarado, P. \& Esteve-Raventós, F. 2017. El género Psathyrella (Fr.) Quél. s.l. en España. VI. Especies nuevas o raras y reevaluación de otras. Bol. Soc. Micol. Madrid 41: 71-98. (SisM, Psathyrella, $\mathrm{CR}, \mathrm{Ge}, \mathrm{Gu}, \mathrm{M}, \mathrm{Na}, \mathrm{O}, \mathrm{Sg})$.

2741. Martínez-Gil, R. \& Martínez, F. 2017. Ascomicetos raros o interesantes de La Rioja, España (III). Bol. Micol. FAMCAL 12: 67-89. (Flora, Ascomycetes, Lo).

2742. Montes, N., Reina, R., Ortiz, T., Ocampo, J.A., García-Romera, I. \& Aranda, E. 2017. Interactions between phenolic compounds present in dry olive residues and the arbuscular mycorrhizal symbiosis. Mycol. Progress 16(6): 567-575. (Ecol, Glomeromycota, Gr). doi: 10.1007/s11557-017-1293-1.

2743. Moreno, G. 2017. In Memoriam. Margarita de la Torre Martín. Bol. Soc. Micol. Madrid 41: 7-9. (Biog).

2744. Moreno, G. 2017. In Memoriam. Leopoldo Miguel Oltra Altisent. Bol. Soc. Micol. Madrid 41: 13-16. (Biog).

2745. Moreno, G., Castillo, A., López-Villalba, A. \& Sánchez, A. 2017. A new species of Hemitrichia in succulent plants. Bol. Soc. Micol. Madrid 41: 31-35. (Tax, Hemitrichia, Al, Ba, Ca, Sg).

2746. Muñoz, G. \& Rojo, C. 2017. Contribución al conocimiento del género Psathyrella en la Península Ibérica (III): Psathyrella epimyces. Bol. Micol. FAMCAL 12: 101107. (Tax, Psathyrella, Sg).

2747. Niell, M. 2017. Los hongos y los espiritus elementales. Rev. Cat. Micol. 38: 59-69. (Etnobot, Amanita, Fomes).

2748. Niell, M., Valverde, A. \& Girbal, J. 2017. Addicions al catàleg micològic d`Andorra. I. Rev. Cat. Micol. 38: 2130. (Flora, Basidiomycetes, And).

2749. Olariaga, I., Corriol, G., Salcedo, I. \& Hanssen, K. 2016. A new species of Typhula with sigmoid spores: Typhula suecica. Karstenia 56: 27-38. (Tax, Typhula, $\mathrm{Hu}, \mathrm{SS}$ ).

2750. Olariaga, I., Moreno, G., Manjón, J.L., Salcedo, I., Hofstetter, V., Rodríguez, D. \& Buyck, B. 2017. Cantharellus (Cantharellales, Basidiomycota) revisited in Europe through a multigene phylogeny. Fungal Diversity 83(1): 263-292. (SisM, Cantharellus, Esp). doi: 10.1007/s13225-016-0376-7. 
2751. Parra, L.A. \& Caballero, T. 2017. Agaricus pietatis, una especie nueva de Agaricus sect. Minores encontrada en España. Bol. Micol. FAMCAL 12: 137-143. (Tax, Agaricus, Lo).

2752. Parra, L.A., Muñoz, G., Pérez-delAmo, F., Martínez, F., Martínez, R. \& Meléndez, A. 2017. Semblanza de nuestro querido amigo Agustín Caba1lero Moreno. Bol. Micol. FAMCAL 12: 11-20. (Biog).

2753. Paz, A., Bellanger, J.-M., Lavoise, C., Molia, A., Ławrynowicz, M., Larsson, E., Ibarguren, I.O., Jeppson, M., Læssøe, T., Sauve, M., Richard, F. \& Moreau, P.-A. 2017. The genus Elaphomyces (Ascomycota, Eurotiales): a ribosomal DNA-based phylogeny and revised systematics of European 'deer truffles'. Persoonia 38: 197-239. (SisM, Astraeus, Elaphomyces, $\mathrm{Cc}, \mathrm{O}, \mathrm{S}, \mathrm{Sg})$. doi: https:// doi.org/10.3767/003158517X697309.

2754. Pedraja Lombillas, S. 2017. Coltricia perennis (L.) Murril J. Mycol. 9(2): 91 (1903). Yesca 29: 61-66. (Ecol, Coltricia, S).

2755. Pérez-de-Gregorio, M.À. \& Sánchez, L. 2017. Tres interessants Mycena trobades a Cataunya. Rev. Cat. Micol. 38: 41-47. (Tax, Mycena, Esp).

2756. Pérez-de-Gregorio, M.A., Sánchez, L. \& Gibert, S. 2017. Mycena plumipes (Kalchbr.) P.-A. Moreau, en España. Yesca 29: 105-110. (Flora, Mycena, $\mathrm{Ge})$.

2757. Pérez-Puente, A. 2017. Algunas especies del género Cortinarius poco frecuentes, recolectadas en (España). Yesca 29: 67-76. (Flora, Cortinarius, $\mathrm{Cs}, \mathrm{Ge}, \mathrm{J}, \mathrm{S})$.

2758. Requejo, O. \& Garea, M. 2017. Algunos hongos raros o poco conocidos en Galicia (N.O. Península Ibérica). Yesca 29: 77-84. (Flora, Basidiomycetes, Or, Po).

2759. Ríos, S., Bautista, A., Verde, A. \& Fajardo, J. 2017. Nueva cita para la micobiota de la Comunidad Valenciana: Verpa bohemica. Bol. Soc. Micol. Madrid 41: 43-46. (Tax, Verpa, A).

2760. Ruiz Mateo, A. 2017. Identificación de las especies del género Parasola presentes en la Península Ibérica por sus caracteres no delicuescentes. Bol.
Micol. FAMCAL 12: 125-136. (Tax, Parasola, Na, So, Z).

2761. Salom, J.C. \& Siquier, J.L. 2017. Leucocoprinus heinemannii, una especie alóctona, antropófila y poco citada, encontrada en Mallorca (Illes Balears, España). Bol. Micol. FAMCAL 12: 5560. (Tax, Flora, Leucocoprinus, Mll).

2762. Sánchez-Pujante, P.J., Miras-Moreno, B., Soluyanova, P., Garre, V., Pedreño, M.A. \& Almagro, L. 2017. Production of fatty acid methyl esters and other bioactive compounds in elicited cultures of the fungus Mucor circinelloides. Mycol. Progress 16(5): 507-512. (Quim, Mucor, Mu). doi: 10.1007/ s11557-017-1278-0.

2763. Sánchez-Sánchez, L., Mateos, F.J. \& Velasco, J.M. 2017. Craterocolla cerasi (Sebacinales, Basidiomycota), una especie muy rara encontrada en la Península Ibérica. Bol. Micol. FAMCAL 12: 91-99. (Tax, Craterocolla, B, Le).

2764. Siquier, J.L., Salom, J.C., Planas, J. \& Llistosella, J. 2017. Contribució al coneixement micolàgic de les Illes Balears (Espanya). XXIII. Mallorca. Rev. Cat. Micol. 38: 1-19. (Flora, Ascomycetes, Basidiomycetes, M11).

2765. Siquier, J.L., Salom, J.C., Planas, J., Espinosa, J., Llistosella, J. \& Serra, A. 2017. Notes corològiques sobre la funga d'Eivissa (Ille Balears, Espanya). IV. Rev. Cat. Micol. 38: 49-57. (Flora, Ascomycetes, Basidiomycetes, Ib).

2766. Suárez, E. \& Suárez Gracía, D. 2017. Adiciones al catálogo corológico de los macromicetos de la provincia de Teruel VI. Bol. Soc. Micol. Madrid 41: 99-115. (Flora, Ascomycetes, Basidiomycetes, Te).

2767. Traba Velay, J.M. 2017. Russula violeipes var. citrina (Quél.) Sarnari f. inolens Traba. Yesca 29: 93-104. (Tax, Russula, O, Vi).

2768. Trapiello, E., Schoebel, C.N. \& Rigling, D. 2017. Fungal community in symptomatic ash leaves in Spain. Baltic Forestry 23(1): 98-73. (Fitopat, SisM, Ascomycetes, O).

2769. Valverde-Valera, A. 2017. Primera aproximació al component fúgic dels voltants de l'Estany de Banyoles. Rev. Cat. Micol. 38: 31-39. (Flora, Ascomycetes, Basidiomycetes, Ge). 
2770. Yurkewich, J.Y., Castaño, C. \& Colinas, C. 2017. Chestnut red stain: Identification of the fungi associated with the costly discolouration of Castanea sativa. Forest Pathology 47(4): 1-9. (Patol, Ascomycetes, Basidiomycetes, B). doi: 10.1111/efp.12335.

\section{Índice temático}

Anatomía (Anat): 2735.

Bibliografía (Bibl): 2721.

Biografías (Biog): 2743, 2744, 2752.

Ecología (Ecol): 2723, 2734, 2736, 2742, 2754.

Etnobotánica (Etnobot): 2747.

Fitopatología (Fitopat): 2768.

Fitoquímica (Quim): 2736, 2762.

Flora (Flora): 2722, 2724, 2733, 2741, 2748, 2756, 2757, 2758, 2761, 2764, 2765, 2766,2769 .

\section{Índice taxonómico}

Agaricus: 2751.

Amanita: 2747.

Ascomycetes: 2741, 2764, 2765, 2766, 2768, 2769,2770 .

Astraeus: 2753.

Basidiomycetes: 2722, 2748, 2758, 2764, 2765, 2766, 2769, 2770.

Cantharellus: 2750.

Clypeophysalospora: 2737.

Coltricia: 2754.

Coniella: 2725.

Cortinarius: 2719, 2758.

Coprinopsis: 2728.

Craterocolla: 2763.

Dendrocorticium: 2739.

Didymium: 2720.

Elaphomyces: 2753.

Fomes: 2747.

Glomeromycota: 2742.

Gyroporus: 2729.

Hemitrichia: 2745.

Hymenochaete: 2726.
2771. Zamora-Ballesteros, C., Haque, M.M.U., Diez, J.J. \& Martín-García, J. 2017. Pathogenicity of Phytophthora alni complex and P. plurivora in Alnus gluinosa seedlings. Forest Pathology 47(2): 1-8. (Patol, Phytophthora, Le). doi: 10.1111/efp.12299.

Patología (Patol): 2770, 2771.

Sistemática (Tax): 2720, 2732, 2739, 2745, 2746, 2749, 2755, 2759, 2760, 2761, 2763, 2767.

Sistemática Molecular (SisM): 2719, 2725, 2726, 2727, 2728, 2729, 2730, 2731, 2737, 2738, 2740, 2750, 2753, 2768.

Hyphoderma: 2732.

Hypholoma: 2723.

Inocybe: 2730.

Inomidotis: 2733.

Leucocoprinus: 2761.

Mucor: 2762.

Mycena: 2755, 2756.

Myriostoma: 2724.

Parasola: 2760.

Paxillus: 2735.

Penicillium: 2731.

Phlebia: 2732.

Phytophthora: 2771.

Preussia: 2738.

Psathyrella: 2740, 2746.

Ramaria: 2727.

Russula: 2767.

Serpula: 2734.

Tuber: 2736.

Typhula: 2749.

Verpa: 2759. 
Índice geográfico

Álava (Vi): 2767.

Alicante (A): 2759.

Huesca $(\mathrm{Hu}): 2749$.

Almería (Al): 2745.

Jaén (J): 2757.

Asturias (O): 2729, 2740, 2753, 2767, 2768.

León (Le): 2763, 2771.

Ávila (Av): 2720.

Madrid (M): 2726, 2730, 2740.

Baleares (PM):

Málaga (Ma): 2739.

Murcia (Mu): 2762.

Ibiza (Ib): 2765.

Mallorca (M11): 2761, 2764.

Badajoz (Ba): 2745.

Barcelona (B): 2719, 2722, 2763, 2770.

Cáceres (Cc): 2727, 2729, 2753.

Cádiz (Ca): 2727, 2745.

Cantabria (S): 2723, 2733, 2753, 2754, 2757.

Castellón (Cs): 2736, 2757.

Ciudad Real (CR): 2740.

Coruña, La (C): 2734.

Gerona (Ge): 2740, 2756, 2757, 2769.

Granada (Gr): 2742.

Guadalajara (Gu): 2728, 2729, 2740.

Navarra (Na): 2740, 2760.

Orense (Or): 2758.

Pontevedra (Po): 2724, 2758.

Rioja, La (Lo): 2741.

Salamanca (Sa): 2732.

Segovia (Sg): 2729, 2740, 2745, 2746, 2753.

Soria (So): 2760 .

Tarragona (T): 2731.

Teruel (Te): 2736, 2766.

Toledo (To): 2726, 2732.

Zamora (Za): 2727.

Zaragoza (Z): 2760.

Guipúzcoa (SS): 2726, 2749.

Huelva (H): 2720, 2725.

ANDORRA (And): 2748.

ESPAÑA (Esp): 2738, 2750, 2755.

PORTUGAL: 2737. 